\title{
Effect of Heat Treatment on Cohesion of Films on Alkali-Treated Titanium
}

\author{
Sengo Kobayashi, Tetsuya Inoue* and Kiyomichi Nakai \\ Department of Materials Science and Engineering, Ehime University, Matsuyama 790-8577, Japan
}

The cohesiveness of films on titanium was evaluated by a scratch test. The films were formed on titanium by immersion in an aqueous solution of $\mathrm{NaOH}$ (hereafter referred as "alkali-treated") followed by heating at $500-700^{\circ} \mathrm{C}$ for $3.6 \mathrm{ks}$. The cohesion of the alkali-treated film was increased by the heat treatment, and the maximum cohesion was obtained by heating at $500^{\circ} \mathrm{C}$. The film on alkali- and heat-treated titanium consisted of both microporous films and dense films. The microporous film furthest from the titanium surface was barely cohesive, whereas the dense film showed cohesion with titanium. The cohesion of film formed by alkali-treatment was increased on heating, but the formation of $\mathrm{TiO}_{2}$ (rutile) at the film-titanium interface on heating at and above $600^{\circ} \mathrm{C}$ caused a deterioration in the cohesion.

(Received October 25, 2004; Accepted January 13, 2005)

Keywords: titanium, alkali treatment, cohesion, scratch test, interface structure, transmission electron microscopy

\section{Introduction}

Titanium covered by hydroxyapatite (HAp) can be directly fused to a living bone after implantation. Kokubo et al. reported that HAp can be chemically coated onto a titanium surface by sequential immersion in aqueous $\mathrm{NaOH}$ solution, heat treatment, and soaking in a simulated body fluid (SBF). ${ }^{1-4)}$ They also confirmed that HAp can be formed in vivo on alkali- and heat-treated titanium. Nishiguchi et al. showed, however, that alkali-treated titanium without heat treatment had no bone-bonding ability in vivo because of delamination of the bioactive film. ${ }^{5)}$ They suggested that both alkali and heat treatment are essential for preparing bioactive titanium. Their results imply that the cohesion of the film on alkali-treated titanium can be increased by heat treatment. Kim et al. examined the cohesion of a HAp layer on alkaliand heat-treated titanium by tensile testing with loading normal to the HAp-titanium interface. ${ }^{6)}$ Because of the lack of adhesion of the glue used to attach the specimen, the effect of heat treatment on cohesion of the HAp layer on alkalitreated titanium was not clarified. Fracture during tensile testing did not occur at the HAp-titanium interface, but at the HAp-glue boundary. Because a film on alkali- and/or heattreated titanium in vivo would be in contact with living bone and be subjected to a shear stress from it, a scratch test appears to be more appropriate than a tensile test for evaluating the cohesion of the film. Scratch tests are increasingly used to evaluate film cohesion. ${ }^{7-10)}$ A scratch is made by a stylus drawn over the specimen's surface. The load for scratching is increased either stepwise or continuously until the film becomes detached from substrate. The load at the moment of detachment of the film is called the critical load and gives a comparative value of film cohesion. ${ }^{7)}$ It is important to clarify the difference in the cohesion of films on alkali- and/or heat-treated titanium, because the formation of HAp on alkali- and/or heat-treated titanium under frictional stress, for example, in vivo, is influenced by the cohesion of the film.

In the present study, the effect of heat-treatment on the cohesion of film on alkali-treated titanium was examined by using the scratch test and was evaluated precisely.

*Graduate student, Ehime University

\section{Experimental Procedures}

A pure sample of titanium was polished by using $0.05 \mu \mathrm{m}$ size alumina powder and then washed sequentially with acetone and purified water in an ultrasonic bath. The sample was then immersed in $5 \mathrm{M}$ aqueous $\mathrm{NaOH}$ solution at $60^{\circ} \mathrm{C}$ for $86.4 \mathrm{ks}$. Following this treatment, the sample was washed gently with purified water, dried at $40^{\circ} \mathrm{C}$ for $86.4 \mathrm{ks}$, heated at $500-700^{\circ} \mathrm{C}$ for $3.6 \mathrm{ks}$ in air, and cooled in the furnace. After alkali-treatment, a microporous sodium titanate hydrogel film was formed on the titanium and this was converted to a microporous structure by dehydration through heat treatment. $^{2,11)}$ The scratch test was carried out by using a HEIDON-14D surface-property tester at room temperature. At least three sections were tested for each specimen prepared under different conditions. The indenter has a conical shape with a spherical sapphire tip with a radius of curvature of $50 \mu \mathrm{m}$. The frictional force was measured as a function of load from 5 to $50 \mathrm{~g}$. The scratching velocity was changed from $8.3 \times 10^{-5}$ to $8.3 \times 10^{-4} \mathrm{~m} / \mathrm{s}$. Because the frictional force of scratching was not sensitive to the scratching velocity in the present study, the scratching velocity was fixed at $1.7 \times 10^{-4} \mathrm{~m} / \mathrm{s}$. The cohesion of the film was evaluated from the critical load $\left(L_{\mathrm{c}}\right)$ obtained from the scratch test. By increasing the load, it was possible to define the $L_{\mathrm{c}}$ for cohesive failure of the film-titanium system. The cohesive failure of the film could be detected by a sudden change in the frictional force during scratching and by microscopic observation of the scratch track. ${ }^{7)}$ The surface morphology and scratch track were examined by using a JSM-5310 scanning electron microscope (SEM). Microstructures at and around the film-titanium interface were examined by transmission electron microscopy (TEM). Thin foils for TEM were prepared by using a GATAN-DuoMill600 ion-milling equipment, and examined in a JEM2000EX TEM operated at $200 \mathrm{kV}$.

\section{Results and Discussion}

Figure 1 shows the variation in frictional force with the load during scratching of titanium. The frictional force increased proportionally with increasing load. The solid line along the data points was drawn by the least-squares method and shows that the relationship between the frictional force 


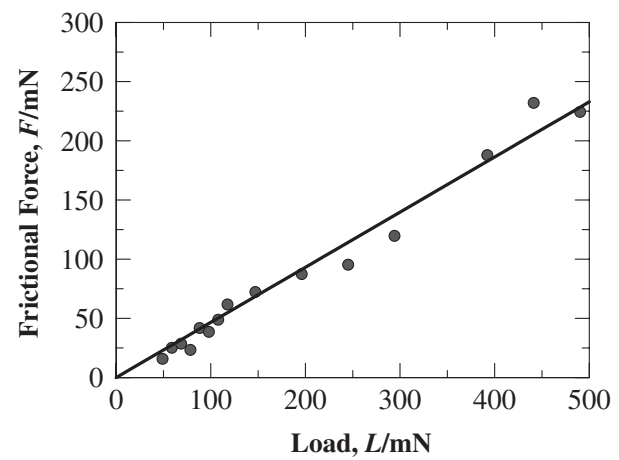

Fig. 1 Variation of frictional force with load for a scratch test of titanium.

$(F)$ and the load $(L)$ is $F=0.466 \times L$. The frictional coefficient is usually defined as $(F / L)$. The value of 0.466 , therefore, represents the frictional coefficient between the sapphire stylus and the titanium substrate. Figure 2(a) shows the variation of the frictional force with load during scratching of alkali-treated titanium. Until the load attained $108 \mathrm{mN}$, the frictional force increased proportionally with the load, whose frictional coefficient was 0.464. At the load of $108 \mathrm{mN}$, the frictional force abruptly increased and the frictional coefficient also increased. Above a load of $108 \mathrm{mN}$, the frictional force was proportional to the load, whose frictional coefficient was 0.712 . The $L_{\mathrm{c}}$ of the film on alkalitreated titanium was $108 \mathrm{mN}$. An increase in the frictional coefficient from 0.464 to 0.712 at the $L_{\mathrm{c}}$ was attributed to the delamination of the film during scratching. Figures $2(\mathrm{~b})-(\mathrm{d})$ show the variation of the frictional force with load during scratching of alkali-treated titanium samples subjected to heat treatment at 500,600 , and $700^{\circ} \mathrm{C}$, respectively. The result for alkali-treated titanium shown in Fig. 2(a) is referred to by dotted lines in Figs. 2(b)-(d). In a specimen heated to $500^{\circ} \mathrm{C}$, the frictional force initially increased with load in a similar manner to that of alkali-treated titanium, and then deviated from it. The $L_{\mathrm{c}}$ of the specimen heated at $500^{\circ} \mathrm{C}$ was $235 \mathrm{mN}$. The frictional coefficient changed from 0.451 to 0.949 at the $L_{c}$. The frictional coefficient of a specimen heated at $600^{\circ} \mathrm{C}$, on the other hand, exhibited a lower value of 0.114 compared with that observed in alkali-treated titanium [Fig. 2(a)]. The $L_{\mathrm{c}}$ of the specimen heated at $600^{\circ} \mathrm{C}$ was $200 \mathrm{mN}$. The frictional coefficient changed from 0.114 to 0.365 at the $L_{\mathrm{c}}$. The variation of the frictional force with load for the specimen heated at $700^{\circ} \mathrm{C}$ exhibited almost the same tendency as that observed in the specimen heated at $600^{\circ} \mathrm{C}$. The $L_{\mathrm{c}}$ of the specimen heated at $700^{\circ} \mathrm{C}$ was $174 \mathrm{mN}$.

Figures 3(a)-(c) and (d)-(f) are SEM and optical micrographs, respectively. They show the scratch track made on alkali-treated specimens heat-treated at $500^{\circ} \mathrm{C}$ under loads of $69 \mathrm{mN}$ [Figs. 3(a) and (d)], $147 \mathrm{mN}$ [Figs. 3(b) and (e)] and $294 \mathrm{mN}$ [Figs. 3(c) and (f)]. Under a load of $69 \mathrm{mN}$, the microporous film on the scratch track was flaked off [Fig. 3(a)]. Such flaking of microporous films was detected even under the smallest load $(49 \mathrm{mN})$. The same type of film failure was also observed under a load of $147 \mathrm{mN}$ [Fig. 3(b)]. Interference fringes on the scratch track observed in Fig. 3(e) indicates that a thin dense film still remains on the scratch track. This result suggests that the film on alkali-treated titanium heat-treated at $500^{\circ} \mathrm{C}$ is layered by a microporous film and a thin dense film. The surface of the scratch track under a load of $147 \mathrm{mN}$ appeared to be rather flat. Above loads of $294 \mathrm{mN}$, on the other hand, the scratch track exhibited some grooves. Such grooves were also observed on a scratch track of a titanium substrate under all the loads examined. The scratch test at the $L_{\mathrm{c}}, 235 \mathrm{mN}$ [Fig. 2(b)], corresponds to a situation between that shown in Figs. 3(b) and (c). At the $L_{\mathrm{c}}$, the dense film will be delaminated from titanium.

The values of $L_{\mathrm{c}}$ evaluated from Fig. 2 were drawn using a
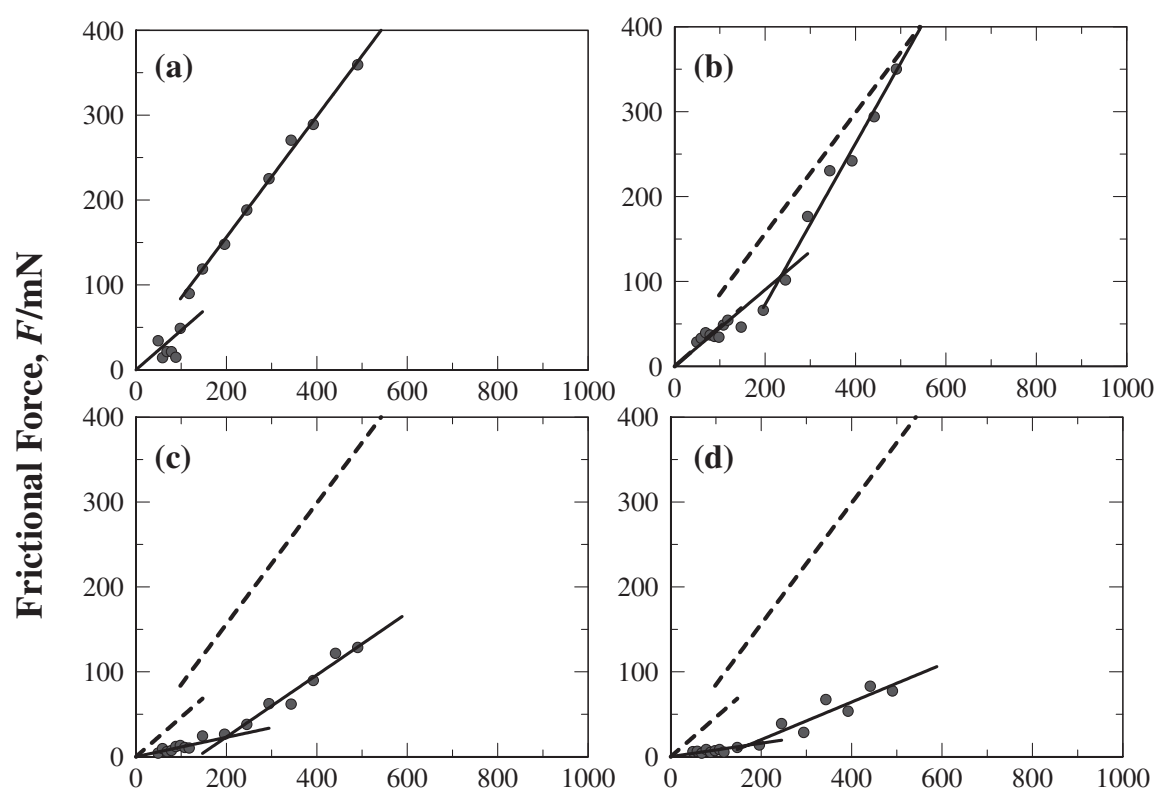

Load, $L / \mathrm{mN}$

Fig. 2 Variation of frictional force with load for scratch tests of films on (a) alkali-treated titanium, and alkali-treated titanium heat treated at (b) 500, (c) 600, and (d) $700^{\circ} \mathrm{C}$. The dashed line in Figs. 2(b)-(d) represents the result for alkali-treated titanium shown in Fig. 2(a). 

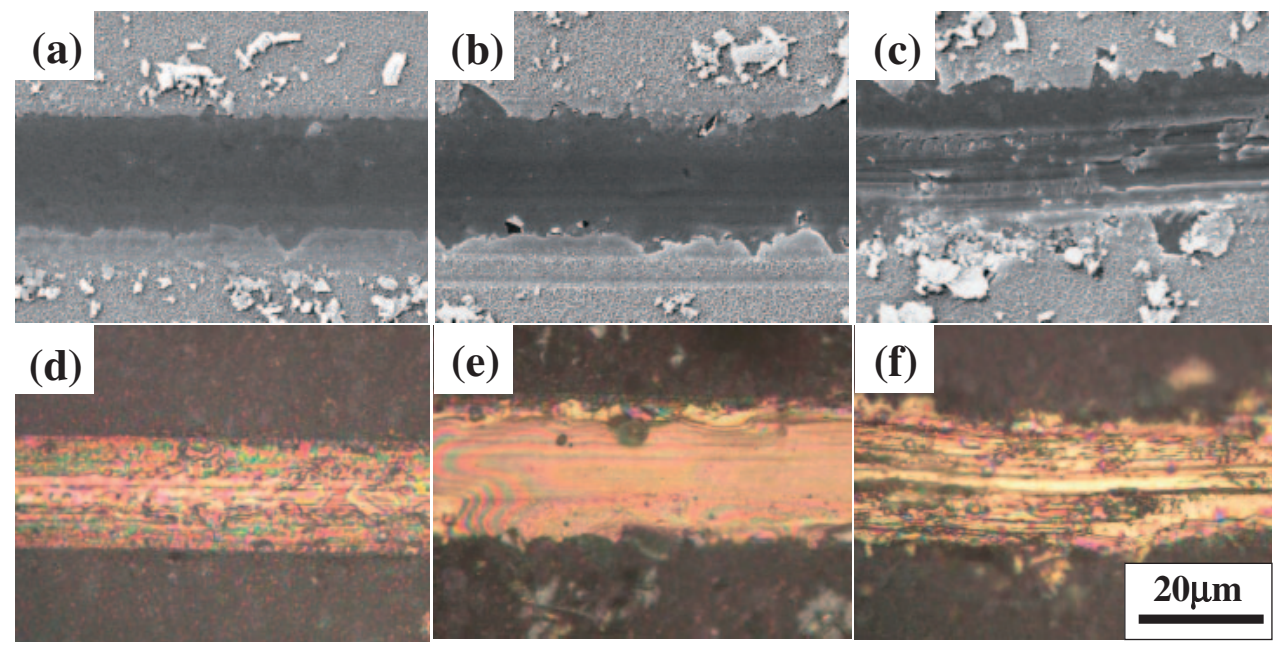

Fig. 3 SEM and optical micrographs showing a scratch track for specimens heated at $500^{\circ} \mathrm{C}$ under loads of $69 \mathrm{mN}$ [(a) and (d)], $147 \mathrm{mN}$ [(b) and (e)] and $294 \mathrm{mN}$ [(c) and (f)].

bar graph in Fig. 4. The $L_{\mathrm{c}}$ of alkali-treated film increased on heating at $500-700^{\circ} \mathrm{C}$. The maximum value of $L_{\mathrm{c}}$ was obtained on heating at $500^{\circ} \mathrm{C}$. The increment in $L_{\mathrm{c}}$ on heating decreased on increasing the heating temperature. The value of $L_{\mathrm{c}}$ in the specimen heated at $500^{\circ} \mathrm{C}$ is about twice compared with that of the alkali-treated specimen, indicating that the cohesion of film on alkali-treated titanium was clearly improved by heating.

The frictional coefficient was clearly decreased by heating above $600^{\circ} \mathrm{C}$, as shown in Figs. 2(c) and (d). The frictional coefficient of a film-titanium system is expressed as $\left(\tau_{\mathrm{f}} / H_{\mathrm{s}}\right)$, where $\tau_{\mathrm{f}}$ and $H_{\mathrm{s}}$ are the shear strength of film and the hardness of titanium, respectively. ${ }^{12)} \mathrm{A}$ Vickers hardness test was performed for all the specimens under a load of $490 \mathrm{mN}$; the results are shown in Fig. 5. The specimen was hardened by heating above $600^{\circ} \mathrm{C}$. The hardness shown in Fig. 5 comprises the hardness of both the film and titanium. Since the hardness of the films is probably almost constant, the titanium itself was hardened by heating above $600^{\circ} \mathrm{C}$, although titanium is generally softened by heating. To clarify the reason for this increase in the hardness of titanium on heating at $600^{\circ} \mathrm{C}$, the microstructure at and near the interface of specimen heated at $600^{\circ} \mathrm{C}$ was examined by TEM. A bright-field TEM image [Fig. 6(a)] shows the microstructure at and near the film-titanium interface for a specimen heated at $600^{\circ} \mathrm{C}$. The diffraction patterns shown in Fig. 6(d)

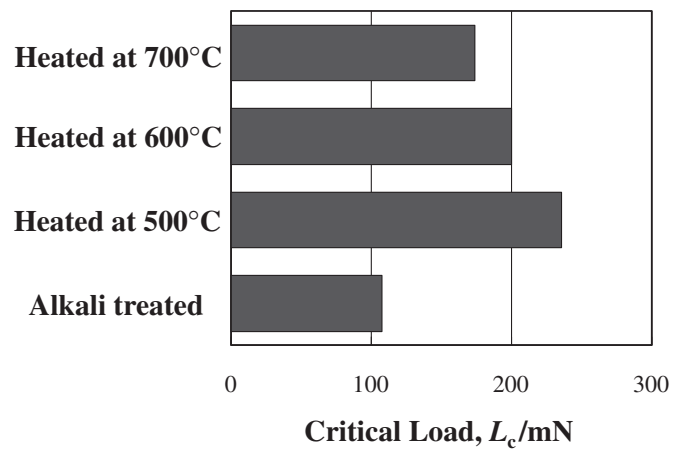

Fig. 4 A bar graph showing the critical load for the specimens examined. revealed that $\mathrm{TiO}_{2}$ and titanium coexisted. The diffraction pattern of $\mathrm{TiO}_{2}$ was indexed as the rutile structure. The darkfield images taken with $(\overline{1} \overline{1} 0)_{\mathrm{TiO}_{2}}$ and $(11 \overline{2} 4)_{\mathrm{Ti}}$ reflections in Figs. 6(b) and (c) show that $\mathrm{TiO}_{2}$ particles with a diameter of about $150 \mathrm{~nm}$ were generated directly on titanium. The layer of $\mathrm{TiO}_{2}$ particles was much thicker (about $8 \mathrm{~nm}$ ) than that usually formed on a titanium surface by natural oxidation, ${ }^{13}$ ) and must, therefore, be formed during the heat treatment. The hardness of $\mathrm{TiO}_{2}\left(\mathrm{Hv} \approx 1121^{14)}\right)$ is much higher than that of titanium $(\mathrm{Hv} \approx 160)$. The increase in hardness on heating at $600^{\circ} \mathrm{C}$ can therefore be attributed to the formation of $\mathrm{TiO}_{2}$. The formation of $\mathrm{TiO}_{2}$ on titanium also occurred in the specimen heated at $700^{\circ} \mathrm{C}$. The film-titanium system on alkali- and heat-treated $\left(>600^{\circ} \mathrm{C}\right)$ titanium is thought to be composed a microporous film, a thin dense film, $\mathrm{TiO}_{2}$, and titanium, starting from the surface. Since the $\tau_{\mathrm{f}}$ in all specimens is probably almost the same, the increase in the hardness of titanium $\left(\Delta H_{\mathrm{s}}\right)$ as a result of the formation of $\mathrm{TiO}_{2}$ decreased the frictional coefficient, $\left(\tau_{\mathrm{f}} / H_{\mathrm{s}}\right)$.

The cohesion of the film on alkali-treated titanium was improved by heating at $500-700^{\circ} \mathrm{C}$. Kim et al. ${ }^{2)}$ suggested that a sodium titanate hydrogel film is formed on titanium immersed in an aqueous $\mathrm{NaOH}$ solution and this is dehydrated and changed into amorphous or crystalline sodium titanate on heating at $500-700^{\circ} \mathrm{C}$. They also indicated that there is a thin region, about $1 \mu \mathrm{m}$ thick, with a compositional gradient of sodium, titanium, and oxygen at

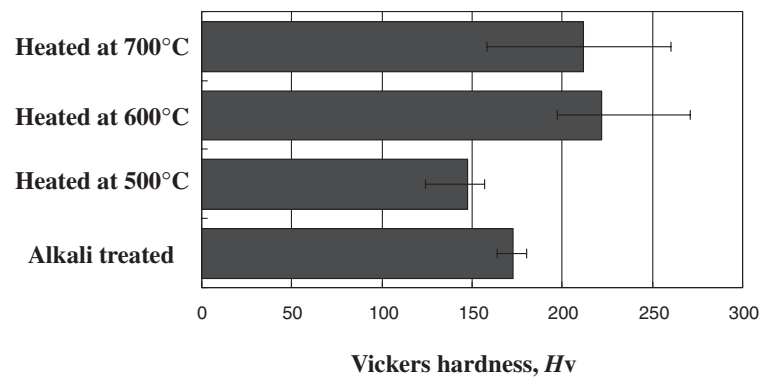

Fig. 5 A bar graph showing the Vickers hardness for the specimens examined. 

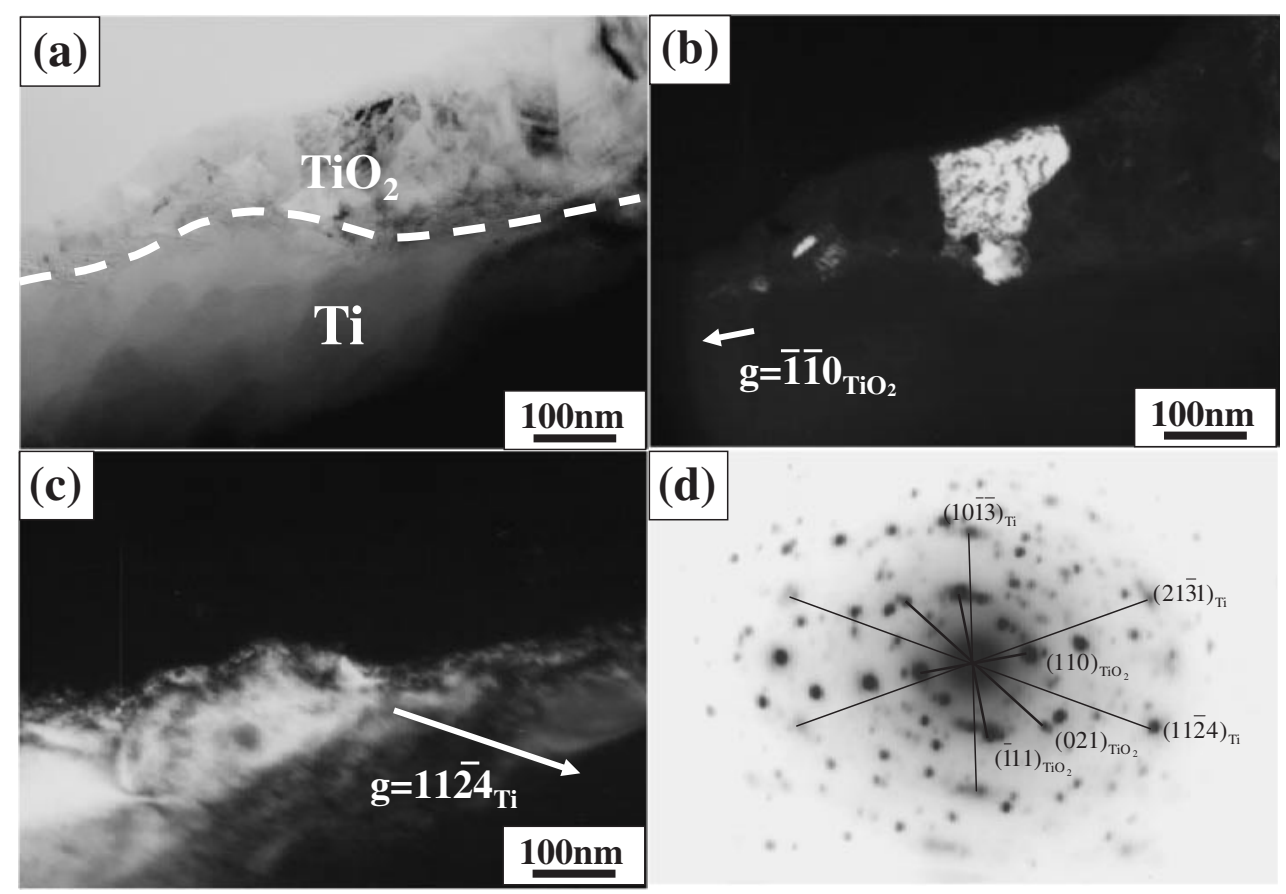

Fig. 6 TEM micrographs for a specimen heated at $600^{\circ} \mathrm{C}$ : (a) bright-field image at and near the interface between the film and titanium substrate, (b) and (c) dark-field image taken with $(\overline{1} \overline{1} 0)_{\mathrm{TiO}_{2}}$ and $(11 \overline{2} 4)_{\mathrm{Ti}}$ reflections and (d) selected area diffraction patterns.

the film-titanium interface. The thin dense film observed on the scratch track shown in Fig. 3(e) could correspond to the thin region of the compositional gradient mentioned above. After heat treatment, amorphous or crystalline sodium titanates, such as $\mathrm{Na}_{2} \mathrm{Ti}_{3} \mathrm{O}_{7}$ and $\mathrm{Na}_{2} \mathrm{Ti}_{6} \mathrm{O}_{13}$, might nucleate in the thin dense film region resulting in the formation of strong bonding with titanium. The increment in the cohesion of the thin film on heating was decreased at temperatures of over $600^{\circ} \mathrm{C}$, probably as a result of the formation of $\mathrm{TiO}_{2}$. Microstructures in the thin film region before and after heat treatment are now under investigation.

\section{Conclusions}

The cohesion of the film on alkali- and/or heat-treated titanium was examined by a scratch test and the results can be summarized as follows.

(1) Heat treatment at temperatures between 500 and $700^{\circ} \mathrm{C}$ improves the cohesion of the film on alkali-treated titanium. The formation of $\mathrm{TiO}_{2}$ above $600^{\circ} \mathrm{C}$, however, causes a deterioration in the cohesion of the film.

(2) The alkali- and/or heat-treated titanium consists, sequentially from the surface, of a microporous film, a thin dense film, $\mathrm{TiO}_{2}$ (on heating above $600^{\circ} \mathrm{C}$ ), and titanium.

(3) The microporous film easily flakes off under scratching. On the other hand, the thin dense film on titanium is resistant to scratching and plays an important role in film cohesion. After heat treatment, the amorphous or crystalline sodium titanate may nucleate in the thin dense film region, resulting in an improvement in film cohesion.

\section{Acknowledgments}

This work was supported in part by a Grant-in-Aid
(No. 14750565) for Scientific Research from the Ministry of Education, Science, Sports, and Culture of Japan. Thanks are also due to the INCS, Ehime University for the use of the transmission electron microscope. The authors wish to express their sincere thanks to Mr. Atsushi Sugino (Nakashima Propeller Co., Ltd.; Nakashima Medical Division) for supplying titanium.

\section{REFERENCES}

1) T. Kokubo, F. Miyaji, H.-M. Kim and T. Nakamura: J. Amer. Ceram. Soc. 79 (1996) 1127-1129.

2) H.-M. Kim, F. Miyaji, T. Kokubo and T. Tanaka: J. Mater. Sci.: Mater. Med. 8 (1997) 341-347.

3) H.-M. Kim, F. Miyaji, T. Kokubo and T. Nakamura: J. Biomed. Mater. Res. 32 (1996) 409-417.

4) T. Kokubo and H.-M. Kim: Materials Integration, 12 (1999) 39-45.

5) S. Nishiguchi, T. Nakamura, M. Kobayashi, H.-M. Kim, F. Miyaji and T. Kokubo: Biomaterials 20 (1999) 491-500.

6) H.-M. Kim, F. Miyaji, T. Kokubo and T. Nakamura: J. Biomed. Mater. Res. 38 (1997) 121-127.

7) J.-F. Lin, C.-C. Wei, Y.-K. Yung and C.-F. Ai: Diam. Relat. Mater. 13 (2004) 1895-1906.

8) M. T. Laugier: J. Mater. Sci. 21 (1986) 2269-2272.

9) N. Laidani, G. Speranza, A. Nefedov, L. Calliari and M. Anderle: Diam. Relat. Mater. 7 (1998) 1394-1402.

10) P. Richard, J. Thomas, D. Landolt and G. Gremaud: Surf. Coat. Technol. 91 (1997) 83-90.

11) S. Kobayashi, K. Kitashita, K. Nakai, N. Kuwano and A. Sugino: 8th Asia-Pacific Conf. on Electron Microscopy, (The Japanese Soc. Microsc., Tokyo, 2004) pp. 761-762.

12) F. P. Bowden and D. Tabor: The Friction and Lubrication of Solids, (Clarendon Press, Oxford, 1986) 112.

13) E. McCafferty and J. P. Wightman: Appl. Surf. Sci. 143 (1999) 92-100.

14) J. F. Shackelford and W. Alexander: CRC Materials Science and Engineering Handbook, 3rd Ed., (CRC Press, Boca Raton, 2001), 474. 